\title{
Discrimination de tempo chez des bébés de 4 mois
}

\section{BARUCH}

Laboratoire de Psychologie Expérimentale, Université René Descartes, Paris V, URA 316 du CNRS, 28 rue Serpente, 75006 Paris, France

\begin{abstract}
résume : The present experiment tested the tempo discrimination abilities of four month-old infants, as a function of tempo range, to investigate whether a similar range of tempo sensitivity is found in infants as in adults. Isochronous auditory sequences were presented with a tempo of 100,300 or $600 \mathrm{~ms}$ inter-onset interval (IOI). Infants were conditioned to associate the auditory sequence and a red light. In an habituation phase, sequences of equal IOI were presented successively, until an habituation criterion was reached. Then for the next three trials, 24 infants (mean age $4 ; 7$ ) heard a sequence with a $15 \%$ faster IOI, and the control group of 15 infants (mean age $4 ; 18$ ) heard the unchanged standard IOI. A reaction to novelty, expressed by longer visual fixations to the light was expected in the experimental group if the two tempi were discriminated. The results show that infants are sensitive to a $15 \%$ IOI increment for habituation IOI of 300 and $600 \mathrm{~ms}$, but not for $100 \mathrm{~ms}$ IOI. Infants discriminate isochronous sequences on the basis of tempo variations, in the same tempo range as adults. These data support the hypothesis that regularity extractor mechanisms are functional at the age of 4 months.
\end{abstract}

Dans sa "théorie de l'attention dynamique", M. Jones et M. Boltz [1] postulent que notre système attentionnel est cyclique, qu'il passe régulièrement par des maxima et des minima d'attention, à une certaine cadence qui pourrait être propre à chaque individu, et qu'elles appellent "période de référence". Les événements se produisant à cette cadence serait par nature mieux traitée qu'à toute autre. $M$. Jones fait l'hypothèse que la période de référence pourrait être liée à des paramètres biologiques, ce qui la conduit à prédire un ralentissement progressif de cette période au fur et à mesure de la vie, du bébé à l'adulte âgé.

Chez l'adulte, des expériences récentes de C. Drake et M.C. Botte [2] ont cherché à déterminer la gamme de tempo à l'intérieur de laquelle la sensibilité à des variations de tempo est la meilleure, en mesurant les seuils différentiels de tempo de séquences isochrones dont les tempi variaient entre 50 et $1500 \mathrm{~ms}$ (ces valeurs de tempo sont en réalité la durée de l'intervalle séparant deux sons successifs (ISD)). En effet, lors d'études plus anciennes, la sensibilité la plus fine avaient été trouvée pour des tempi de $100 \mathrm{~ms}$ [3] ou de $600 \mathrm{~ms}$ [4] selon la recherche. C. Drake et M.C. Botte ont observé une courbe de sensibilité en $U$, avec une zone optimale comprise entre 300 et $800 \mathrm{~ms}$, quelque soit le nombre d'intervalles composant les séquences (de 1 à 6). La sensibilité s'améliore lorsque le nombre d'intervalles augmente, tout en respectant le même pattern. C. Drake \& $M$. Jones [5] en concluent que le système serait "prédisposé" à analyser des régularités, en particulier l'isochronie, et que cette prédisposition ne serait dominante que dans cette zone de tempo, qui pourrait correspondre à la période de référence postulée par M. Jones et al. [1].

L'expérience présentée ici a pour objectif de recueillir de premiers indices permettant d'étayer l'hypothèse selon laquelle les mécanismes permettant la discrimination du tempo serait précocementopérationnels, dès l'âge de 4 mois. On se demandera également si cette discrimination est aussí 
efficace pour différents tempi, ou si au contraire elle serait plus fine pour certains d'entre eux, comme c'est le cas chez l'adulte. Dans ce dernier cas, nous examinerons si les tempi concernés sont les mêmes que chez les adultes, ou plus rapides.

\section{METHODE}

\section{1 stimulus}

Nous avons présenté par haut-parleur aux bébés des séquences régulières composées de sons de cloche brefs ( $50 \mathrm{~ms}$ de période stable), générés par un synthétiseur, de fréquence fondamentale $440 \mathrm{~Hz}$ et de niveau moyen, séparés par des silences de durée variable tesl que l'intervalle séparant les débuts de deux sons successifs (ISD) soit de $100 \mathrm{~ms}, 300 \mathrm{~ms}$, ou $600 \mathrm{~ms}$, testés dans trois conditions expérimentales séparées. Un micro-ordinateur commandait le synthétiseur, et pilotait l'ensemble de l'expérience.

\section{2 sujets}

Le groupe expérimental était composé de 24 enfants (âge moyen 4 mois 8 jours [3;17-5;0]). 11 ont participé aux trois conditions, 11 à deux conditions et 2 à une seule condition. Dans le cas où un même bébé passait successivement plusieurs conditions l'ordre de passation était contrebalancé à l'intérieur de chaque groupe. Le tableau 1 récapitule les effectifs par condition.

Tableau 1. Nombre de bébés par condition.

\begin{tabular}{|llll|}
\hline ISD (ms) & 100 & 300 & 600 \\
\hline groupe expérimental & 17 & 20 & 20 \\
groupe contrôle & 12 & 13 & 14 \\
\hline
\end{tabular}

\section{3 procédure}

La méthode utilisée a été issue de celle développée par L. Demany [6], [7]. Les bébés étaient conditionnés à déclencher la présentation d'une séquence sonore en regardant une cible lumineuse, et à l'arrêter en détournant le regard. L'indice recueilli était le temps de fixation visuel du bébé sur la cible à chaque essai; On procédait parallèllement à la phase d'habituation, puisque à chaque essai successif était présentée une séquence de même tempo, jusqu'à ce qu'une diminution suffisante des temps de fixation ait été enregistrée. On passait alors à la phase test, durant laquelle les séquences étaient présentées avec un tempo $15 \%$ plus rapide. Si les deux tempi étaient discriminés, on s'attendait à observer une réaction à la nouveauté dans cette dernière phase, mise en évidence par une élévation des durées de fixation.

Les bébés étaient installés sur les genoux de leur mère devant un paravent sur lequel était placé, à $45^{\circ}$ à droite du bébé une grosse lampe rouge. un haut-parleur se trouvait derrière le paravent, dans la même direction que la lampe par rapport au regard du bébé. Un expérimentateur se tenait derrière le paravent et observait la direction du regard du bébé au travers d'un petit trou situé sous la lampe. Au démarrage d'un essai, la lampe s'allumait. Lorsque le regard du bébé se posait sur elle, l'expérimentateur déclenchait la présentation de la séquence sonore à un tempo donné. Lorsque le bébé détournait le regard de la lampe, l'expérimentateur arrêtait la séquence et la lampe. L'ordinateur enregistrait le temps de fixation du bébé pendant l'essai. Deux secondes plus tard, la lampe se rallumait et un nouvel essai pouvait commencer.

Durant la phase d'habituation, des séquences de même tempo étaient présentées essai après essai. L'habituation était considérée comme réalisée lorsque la moyenne des temps de fixation visuelle de trois essais consécutifs était inférieure à la moyenne des trois plus longs essais parmi les cinq premiers. Alors était présenté pendant trois essais test un tempo de $15 \%$ plus rapide aux sujets du groupe expérimental $(85 \mathrm{~ms}$ pour une habituation à $100 \mathrm{~ms}, 255 \mathrm{~ms}$ pour $300 \mathrm{~ms}, 510 \mathrm{~ms}$ pour 600 $\mathrm{ms}$ ), et 3 essais au tempo d'habituation aux sujets du groupe contrôle. Les bébés qui, au bout de quinze essais n'avaient toujours pas manifesté d'habituation, ainsi que ceux qui ont pleuré pendant l'expérience ont été exclus de l'étude ( 14 bébés). 


\section{RESULTATS}

A

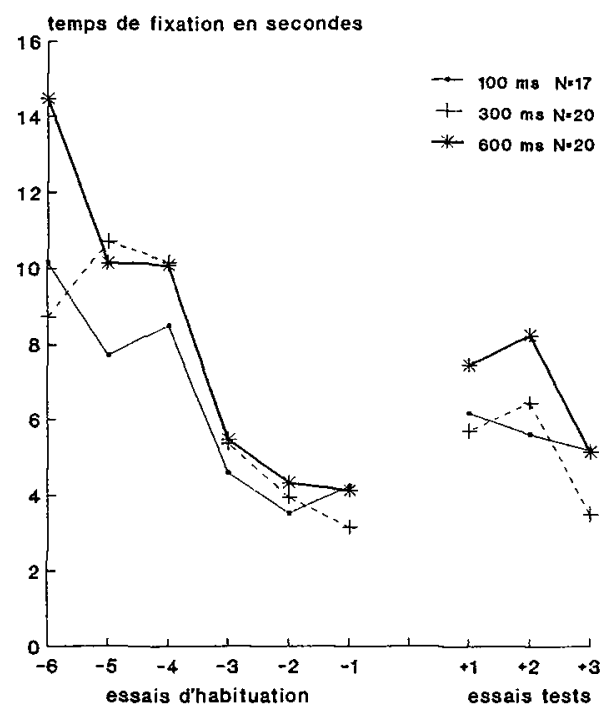

B

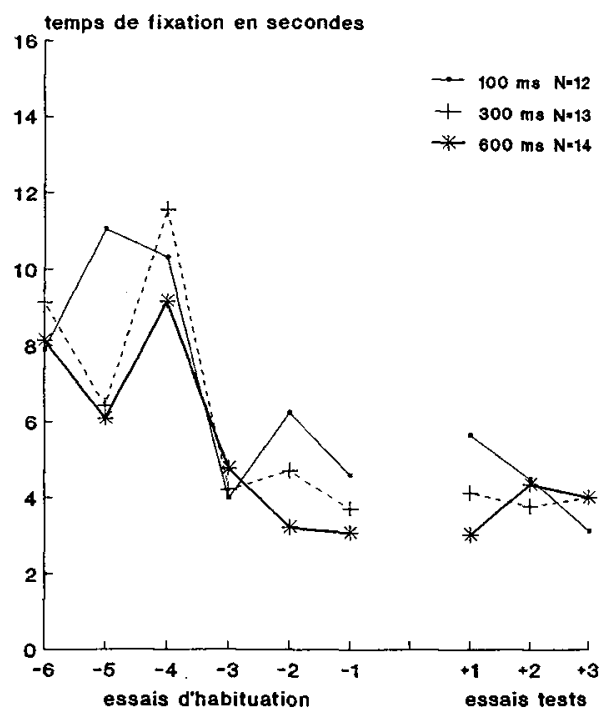

Fig. 1. temps moyens d'observation (en secondes) en fonction des essais. La fig. 1A représente les résultats des groupes expérimentaux, la fig. $1 \mathrm{~B}$ ceux des groupes contrôles. Chaque courbe correspond à l'un des tempi (ISD 100,300 et $600 \mathrm{~ms}$ ).

Les résultats sont présentés figure 1 (1A pour les groupes expérimentaux, 1B pour les groupes contrôles). Les temps moyens d'observation en secondes sont portés en fonction des essais (six derniers essais d'habituation puis 3 essais test) pour chacun des trois tempi (ISD 100, 300 et $600 \mathrm{~ms}$ ).

On peut observer une diminution des temps moyens d'observation au fur et à mesure des essais durant la phase d'habituation, ce qui valide la procédure utilisée; à l'essai (-6), les bébés entendent la séquence en moyenne 10 secondes, tandis qu'à l'essai $(-1)$, le temps de présentation est en moyenne de 3 secondes. Le nombre moyen d'essais nécessaires pour atteindre le critère est de 9.2 pour les groupes expérimentaux et de 9.4 pour les groupes contrôles.

La fig. 1A montre une réaction à la nouveauté lors de la phase test, pour le groupe expérimental, dans les conditions $300 \mathrm{~ms}$ et $600 \mathrm{~ms}$. L'analyse de variance met en évidence une différence significative des temps moyens d'observation entre le dernier essai d'habituation et le premier essai test pour 300 et $600 \mathrm{~ms}(\mathrm{~F}(1-19)=6.79 ; \mathrm{p}<.02$ et $\mathrm{F}(1-19=5.22 ; \mathrm{p}<.03)$, et non pour $100 \mathrm{~ms}(\mathrm{~F}(1-16=1.71, \mathrm{NS})$, même si la tendance va dans le même sens pour les trois tempi. Une variation de $15 \%$ du tempo est discriminable par des bébés de 4 mois dans une gamme de tempi moyens.

Les résultats des groupes contrôles (fig. 1B) ne font apparaître aucune différence entre les derniers essais d'habituation et les essais tests, ce qui confirme que les réactions à la nouveauté observées dans les groupes expérimentaux peuvent bien être imputées à la différence de tempo. Les résultats des groupes expérimentaux et contrôles au premier essai test (essai +1$)$ sont significativement différents pour un tempo de $600 \mathrm{~ms}(\mathrm{~F}(1-32)=5.55 ; \mathrm{p}<.03)$, mais pas pour les ISD 100 , ni $300 \mathrm{~ms}$.

\section{DISCUSSION}

Ces résulats sont les premiers de ce qui devra être une série beaucoup plus approfondie, et donnent quelques indications préliminaires. Ils permettent de considérer que des bébés de 4 mois sont 
capables de discriminer une variation de tempo de $15 \%$ dans une séquence sonore isochrone, après une habituation à $600 \mathrm{~ms}$. Cette valeur correspond à la valeur minimale du seuil différentiel de tempo chez les adultes. Les résultats obtenus pour l'ISD $300 \mathrm{~ms}$ ne sont pas aussi clairs, mais aucune discrimination n'a été mise en évidence pour l'ISD $100 \mathrm{~ms}$. Il est vraisemblable qu'on puisse mettre également en évidence une discrimination à ces tempi en augmentant le pourcentage de variation lors des essais tests.

En conclusion, les mécanismes extracteurs de régularité mis en évidence chez l'adulte semblent déjà opérationnels à 4 mois, dans la même gamme de tempo que chez l'adulte. De nombreuses expériences doivent confirmer ces premiers résultats en testant davantage de tempi différents. Il faut également explorer quels pourcentages de variation de tempo entre phase d'habituation et phase test provoquent des réactions à la nouveauté, pour avoir un indice permettant la comparaison entre la sensibilité des bébés et des adultes. Il est enfin indispensable de répliquer ces expériences avec des enfants plus jeunes, dans l'idéal avec des nouveau-nés, quoique dans ce cas, la méthode utilisée ne soit peut-être pas applicable.

\section{REFERENCES}

[1] Jones M.R. \& Boltz M., dynamic attending and response to time. Psychological Review, 96, 1989, 459-491.

[2] Drake C. \& Botte M.C., Tempo sensitivity in auditory sequences: Evidence for a multiple-look model. Perception \& Psychophysics, 54(3), 1993, 277-286.

[3] Michon J.A., Studies on subjective duration : I- differential senssitivity in the perception of repeating temporal sequences, Acta psychologica, 22, 1964, 441-450.

[4] Fraisse P., Le seuil différentiel de durée dans une suite régulière d'intervalles, Année psychologique, 1, 1967, 43-49.

[5] Drake C. \& Jones M.R., Dynamic attending to auditory sequences : a developmental approach. Comm. soumise au 3rd International Conference for Music Perception and Cognition, Liège, July 1994.

[6] Demany L., Auditory stream segregation in infancy, Infant behavior \& development, 5, 1982, 261276.

[7] Demany L. \& Armand F., The perceptual reality of tone chroma in early infancy, J. Acoust. Soc $A m,(1), 1984,57-66$. 